\title{
Risk of bleeding associated with antiangiogenic monoclonal antibodies bevacizumab and ramucirumab: a meta-analysis of 85 randomized controlled trials
}

This article was published in the following Dove Press journal: OncoTargets and Therapy

\section{Bingkun Xiao \\ Weilan Wang ${ }^{2}$ \\ Dezhi Zhang ${ }^{3}$}

'Department of Pharmaceutical Sciences, Beijing Institute of Radiation Medicine, Beijing, China; ${ }^{2}$ Department of Pharmacy, Chinese PLA General Hospital, Beijing, China; ${ }^{3}$ Department of Pharmacy, The 264th Hospital of PLA, Taiyuan, Shanxi, China
Correspondence: Weilan Wang Department of Pharmacy, Chinese PLA General Hospital, No 28, Fuxing Rd, Beijing 100853, China

Tel +86 I066937243

Email I366I282643@I63.com
Aim: Bevacizumab and ramucirumab are antiangiogenic monoclonal antibodies, which target vascular endothelial growth factor-A and vascular endothelial growth factor receptor-2, respectively, used in various cancers. Bleeding events have been described with these two agents. We conducted an up-to-date meta-analysis to determine the relative risk (RR) associated with the use of antiangiogenic monoclonal antibodies, bevacizumab and ramucirumab.

Methods: This meta-analysis of randomized controlled trials was performed after searching PubMed, American Society for Clinical Oncology Abstracts, European Society for Medical Oncology Abstracts, and the proceedings of major conferences for relevant clinical trials. RR and $95 \%$ CIs were calculated by random-effects or fixed-effects models for all-grade and highgrade bleeding events related to the angiogenesis inhibitors.

Results: Eighty-five randomized controlled trials were selected for the meta-analysis, covering 46,630 patients. The results showed that antiangiogenic monoclonal antibodies significantly increased the risk of all-grade (RR: 2.38, 95\% CI: 2.09-2.71, $p<0.00001$ ) and high-grade (RR: $1.71,95 \%$ CI: $1.48-1.97, p<0.00001)$ bleeding compared with control arms. In the subgroup analysis, bevacizumab significantly increased the risk of all-grade (RR: $2.73,95 \% \mathrm{CI}$ : 2.24-3.33, $p<0.00001$ ) and high-grade bleeding (RR: 1.98, 95\% CI: $1.68-2.34, p<0.00001$ ), but ramucirumab only increased the risk of all-grade bleeding (RR: $1.94,95 \%$ CI: 1.76-2.13, $p<0.00001$ ) and no difference was observed for the risk of high-grade bleeding (RR: $1.04,95 \%$ CI: $0.78-1.39, p=0.79$ ) compared with the control group. For lung cancer patients, bevacizumab significantly increased the risk of all-grade (RR: 4.72, 95\% CI: 1.99-11.19, $p=0.0004$ ) and high-grade pulmonary hemorrhage (RR: $3.97,95 \% \mathrm{CI}: 1.70-9.29, p=0.001$ ), but no significant differences in the risk of all-grade (RR: $1.09,95 \% \mathrm{CI}: 0.76-1.57, p=0.64)$ and high-grade (RR: $1.22,95 \%$ CI: $0.35-4.21, p=0.75)$ pulmonary hemorrhage were observed for ramucirumab. The increased risk of all-grade and high-grade bleeding was also observed in colorectal cancer or non-colorectal tumors and low-dose or high-dose angiogenesis inhibitors.

Conclusion: Antiangiogenic monoclonal antibodies are associated with a significant increase in the risk of all-grade and high-grade bleeding. Ramucirumab may be different from bevacizumab in terms of the risk of high-grade bleeding and the risk of all-grade and high-grade pulmonary hemorrhage in lung cancer patients.

Keywords: bevacizumab, ramucirumab, antiangiogenic monoclonal antibodies, bleeding, meta-analysis 


\section{Introduction}

Angiogenesis is a complex biological process that plays an important role in sustaining growth, invasion, and the metastatic potential of tumors, and this process is mainly driven by vascular endothelial growth factor (VEGF). ${ }^{1,2}$ One of the VEGF family members, VEGF-A (commonly referred to as VEGF), has been demonstrated to be important in angiogenesis. Among all receptors, vascular endothelial growth factor receptor (VEGFR)-2 is widely thought to be principally linked to the stimuli of angiogenesis in malignancies. Blocking the function of VEGF-A or its receptor VEGFR-2 has been the most important antiangiogenic strategy for cancer therapy. ${ }^{3}$

Bevacizumab and ramucirumab are the most important antiangiogenic monoclonal antibodies, which target VEGF-A and its receptor VEGFR-2, respectively, used in various cancers. Bevacizumab is approved by the Food and Drug Administration (FDA) for the treatment of patients with metastatic colorectal cancer, advanced non-squamous non-small cell lung cancer (NSCLC), metastatic renal cell carcinoma, recurrent glioblastoma, advanced cervical cancer, and platinum-resistant ovarian cancer, and ramucirumab is approved by the FDA for the treatment of advanced gastric or gastroesophageal junction adenocarcinoma, metastatic NSCLC, and advanced colorectal cancer.

Bleeding events are a kind of major adverse events reported in clinical trials of bevacizumab and ramucirumab, which may cause severe outcomes that could be even life threatening. ${ }^{4}$ The main mechanism of bleeding is that angiogenesis inhibitors disrupt tumor vasculature through inhibition of VEGF signaling and lead to thrombosis or bleeding. ${ }^{1,5}$

However, the relative risk (RR) of bleeding events in patients with cancer treated with these two antiangiogenic monoclonal antibodies has yet to be defined. Therefore, we conducted an up-to-date meta-analysis of available clinical trials to determine the RR of bleeding in cancer patients treated with antiangiogenic monoclonal antibodies, bevacizumab and ramucirumab.

\section{Materials and methods Search strategy}

This study was conducted in accordance with the guidelines of the Preferred Reporting Items for Systematic Reviews and Meta-Analyses statement ${ }^{6}$ (Supplementary material). We searched PubMed, American Society for Clinical Oncology Abstracts, and European Society for Medical Oncology Abstracts for relevant trials till September 2017. Moreover, we also searched the clinical trial registration website (https://
www.ClinicalTrials.gov) to obtain information on registered randomized controlled trials (RCTs). Keywords used in the search were "bevacizumab," "avastin," "ramucirumab," "IMC1121B," "LY3009806," and "randomized controlled trials." The search was limited to RCTs published in English.

\section{Selection of trials}

Data abstraction and quality assessment were conducted independently by two reviewers. Disagreements were resolved by discussion with an independent expert. The RCTs were eligible for inclusion in our meta-analysis: 1) prospective Phase II and Phase III RCTs in patients with cancer, 2) random assignment of participants to these two antiangiogenic monoclonal antibodies treatment or control groups, 3) available data, including the event or incidence of bleeding and sample size for analysis. Phase I and single-arm phase II trials were excluded because of their lack of control groups.

\section{Data extraction}

We extracted details on study characteristics, treatment information, results, and safety profiles from the selected trials. Clinical endpoints were obtained from the safety profile of each clinical trial. All-grade, high-grade bleeding and all-grade, high-grade pulmonary hemorrhage in lung cancers were recorded according to the version of National Cancer Institute-Common Terminology Criteria for Adverse Events used in each trial.

\section{Statistical analysis}

Data were calculated by Review Manager version 5.2 (The Nordic Cochrane Centre, Copenhagen, Denmark). For the outcomes, the RR was calculated for dichotomous data. Statistical heterogeneity in the results of the trials was assessed by the chi-square test, and expressed by the $I^{2}$ index. ${ }^{7}$ When there was no statistically significant heterogeneity, a pooled effect was calculated with a fixed-effect model. When considerable heterogeneity was found ( $p<0.1$, or $I^{2}>50 \%$ ), a random-effect model was employed. Subgroup analysis was conducted to examine whether the RRs of all-grade and high-grade bleeding varied by drug type, drug dosage, and cancer type.

\section{Results \\ Search results}

We reviewed 2,045 potentially relevant articles from our initial search strategies. A total of 1,906 articles were excluded on screening abstracts and titles for the following reasons: review articles, case reports, basic researches, 
Phase I or single-arm Phase II studies, irrelevant topics, and duplicate reports. The remaining 139 articles were retrieved for full evaluation, and 54 articles were excluded for unavailable data for assessment of bleeding or antiangiogenic monoclonal antibodies in both treatment and control arms. Finally, 85 RCTs were included in this meta-analysis. ${ }^{8-92}$ The study search process is shown in a flow chart (Figure 1).

\section{Patients}

A total of 85 studies and 46,630 patients were included for the analysis. Bevacizumab was investigated in 72 trials $^{8-79}$ and ramucirumab was investigated in 13 trials. ${ }^{80-92}$ All of the studies included 21 colorectal cancer, ${ }^{8-26,85,86} 15$ breast cancer, ${ }^{27-39,87,88} 16$ lung cancer, ${ }^{40-52,80-82}$ three renal cell cancer, ${ }^{53,54}$ two pancreatic cancer, ${ }^{55,56}$ five ovarian cancer, ${ }^{57-61}$ six gastric or gastroesophageal junction adenocarcinoma, ${ }^{62-65}$ ${ }^{89-91}$ three glioblastoma, ${ }^{66-68}$ one lymphoma, ${ }^{69}$ one lymphocytic leukemia, ${ }^{70}$ two melanoma, ${ }^{71,72}$ two malignant mesothelioma, ${ }^{73,74}$ one prostate cancer, ${ }^{75}$ one cervical cancer, ${ }^{76}$ one leiomyosarcoma, ${ }^{77}$ two urothelial carcinoma, ${ }^{83,84}$ two hepatocellular carcinoma, ${ }^{78,92}$ and one soft tissue sarcoma. ${ }^{79}$ In addition, 35 trials ${ }^{9,10,12-20,22-26,46,49,52,55,58,62-65,72,78-84,87,88}$ were treated with low-dose drugs (28 trials for bevacizumab at $2.5 \mathrm{mg} / \mathrm{kg} /$ week, seven trials for ramucirumab at $3.3 \mathrm{mg} / \mathrm{kg} /$ week) and 46 trials ${ }^{11,21,27,28,30-39,41,42,44,45,47,48,50,51,53,54,56,57,59-61,66-71,}$ 73-77,85,86,89-92 were treated with high-dose drugs (40 trials for bevacizumab at $5 \mathrm{mg} / \mathrm{kg} /$ week, six trials for ramucirumab at $4 \mathrm{mg} / \mathrm{kg} /$ week). Other 4 three-arm trials ${ }^{8,29,40,43}$ were two arms of different dosage levels of bevacizumab and one arm of control. All of these RCTs were judged to be of adequate quality (Jadad score is 3-5). Baseline characteristics of the 85 RCTs are provided in Table 1.

\section{RR of all-grade bleeding}

Forty-three RCTs were available to calculate the RR of allgrade bleeding in patients assigned to angiogenesis inhibitors arms versus control arms. The results showed that antiangiogenic monoclonal antibodies significantly increased the risk of all-grade (RR: 2.38, 95\% CI: 2.09-2.71, $p<0.00001$ ) bleeding compared with control arms. There was statistically significant heterogeneity $\left(I^{2}=74 \%\right)$ across the trials; we incorporated it into a random-effects model (Figure 2).

\section{RR of high-grade bleeding}

The RR of high-grade ( $\geq$ grade 3 ) bleeding was determined in 82 RCTs. The results showed that antiangiogenic monoclonal antibodies significantly increased the risk of all-grade bleeding (RR: 1.71, 95\% CI: 1.48-1.97, $p<0.00001)$ with a fixed-effects models $\left(I^{2}=19 \%\right)$ (Figure 3 ).

\section{RR according to drug type}

As an exploratory analysis, patients were stratified according to drug type. We found that bevacizumab significantly increased the risk of all-grade (RR: $2.73,95 \%$ CI: 2.24-3.33, $p<0.00001)$ and high-grade bleeding (RR: 1.98, 95\% CI: $1.68-2.34, p<0.00001)$, but ramucirumab only increased

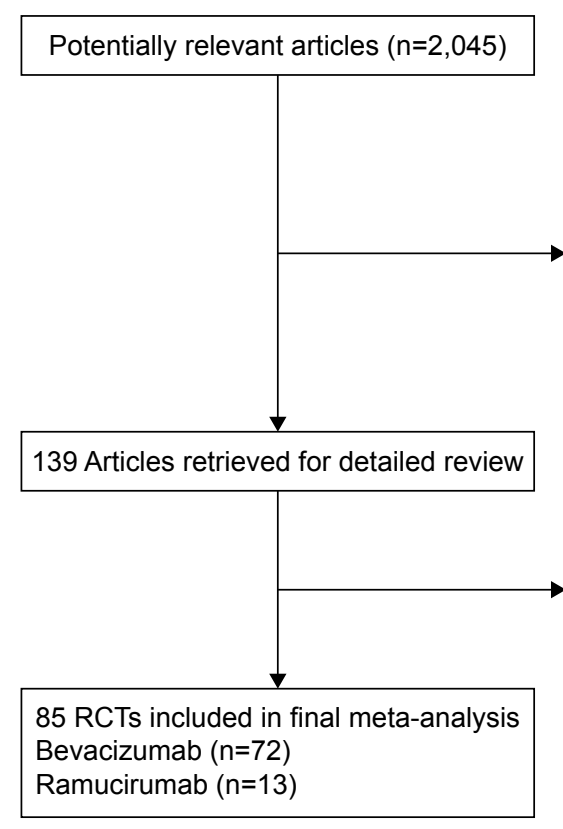

1,906 Articles were excluded after topic or abstract review

Review articles

Case reports

Basic researches

Phase I or single-arm phase II studies

Irrelevant topic

Duplicate reports

54 Articles were excluded due to

Unavailable data for assessment of bleeding

Antiangiogenic monoclonal antibodies in both

treatment and control arms

Figure I Outline of the search flow diagram.

Abbreviation: RCTs, randomized controlled trials. 


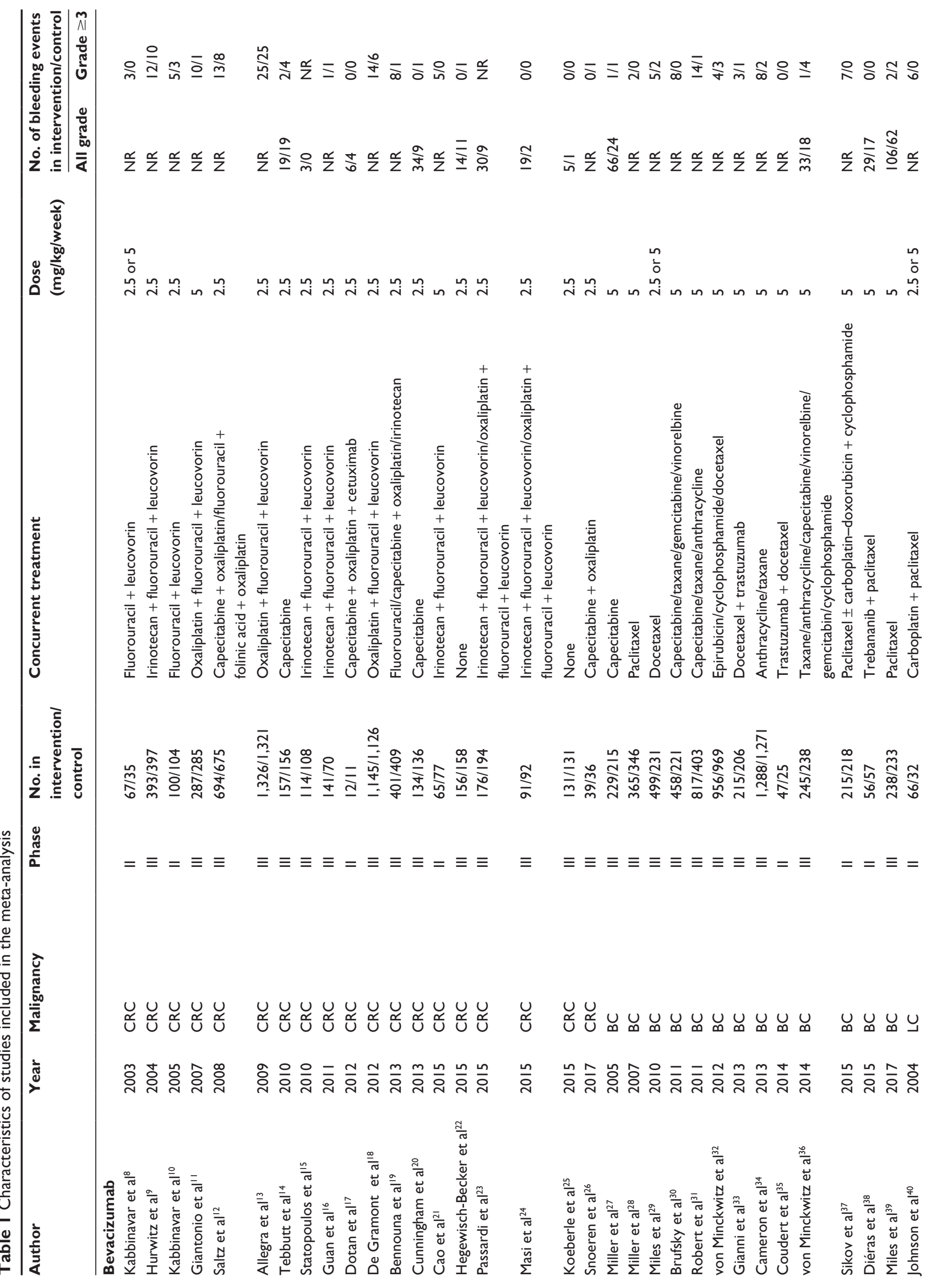




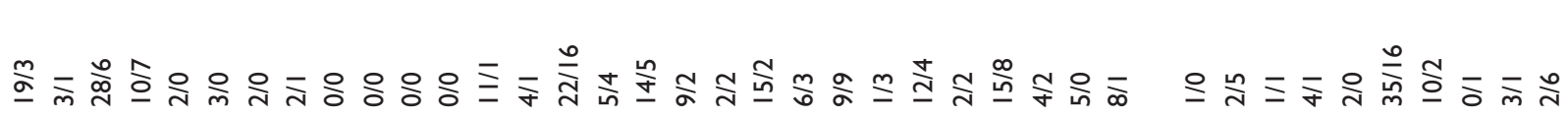

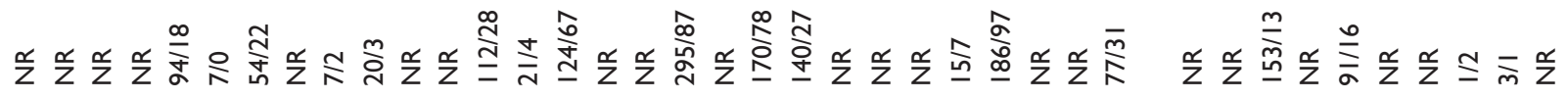

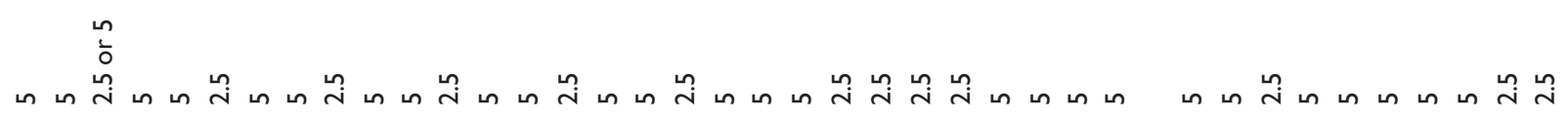

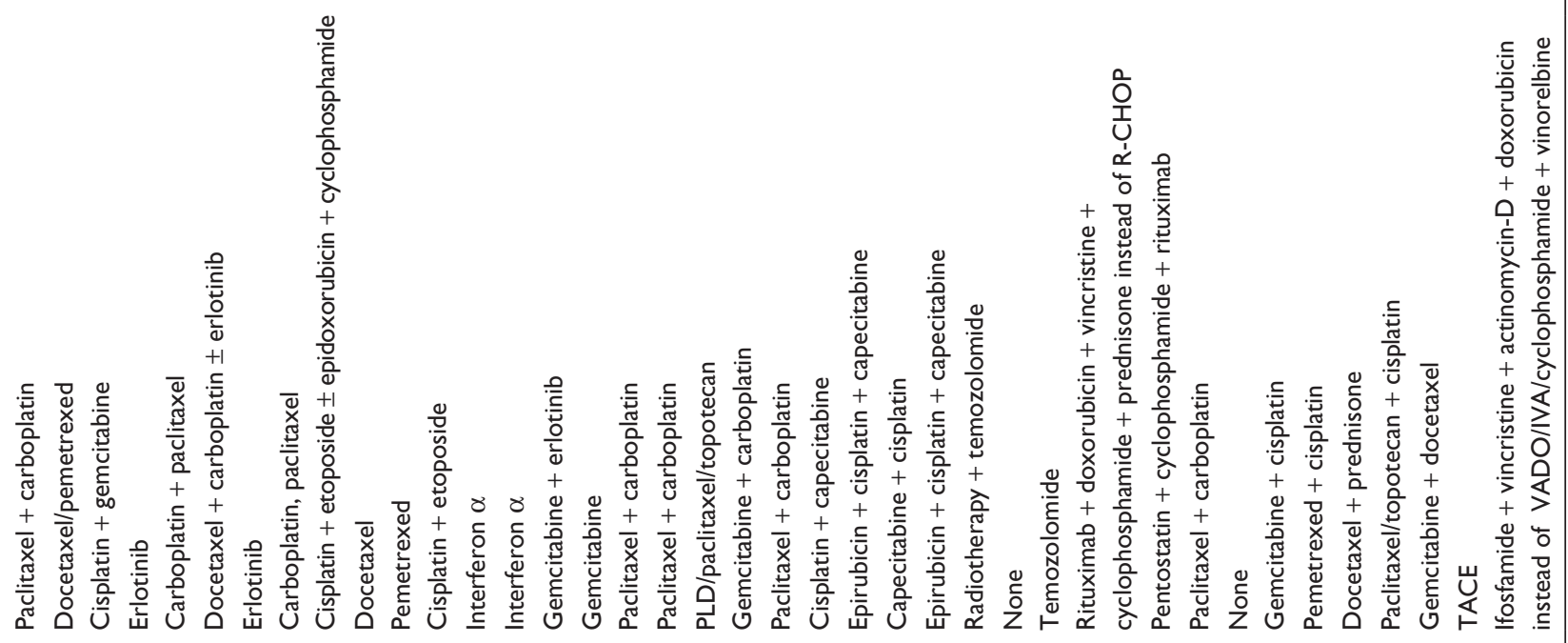

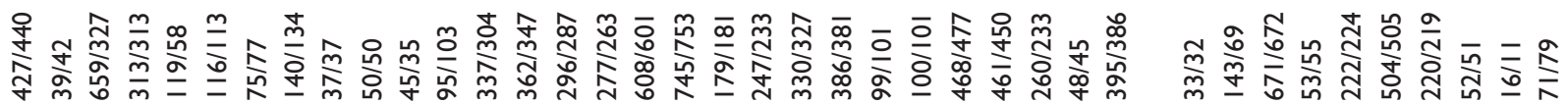

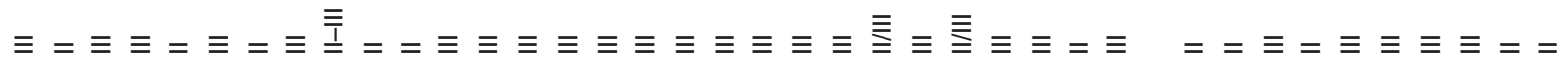

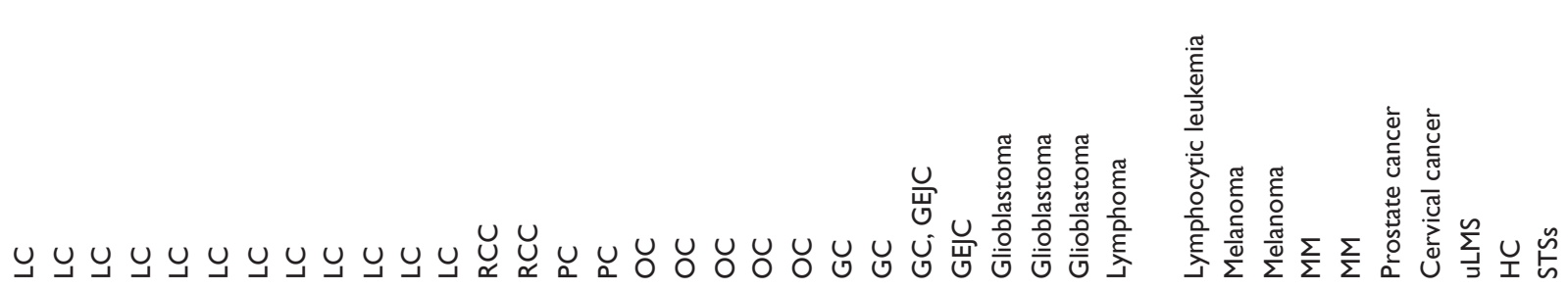

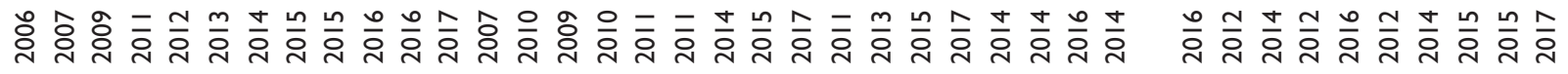

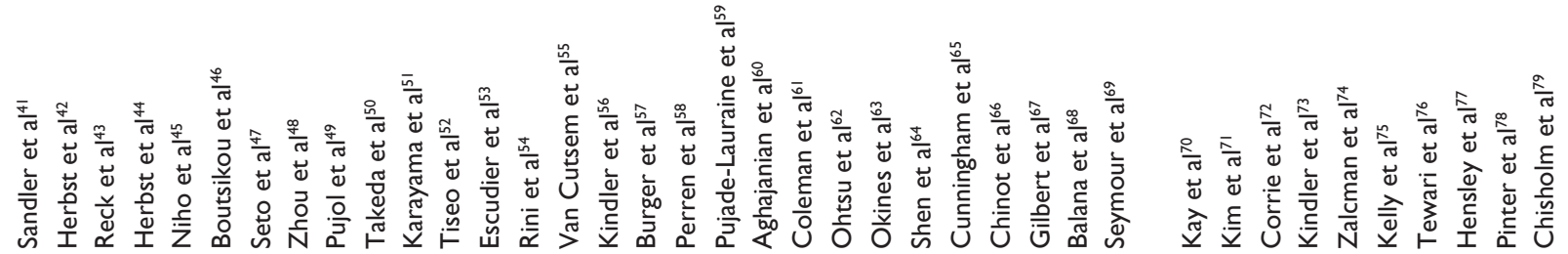




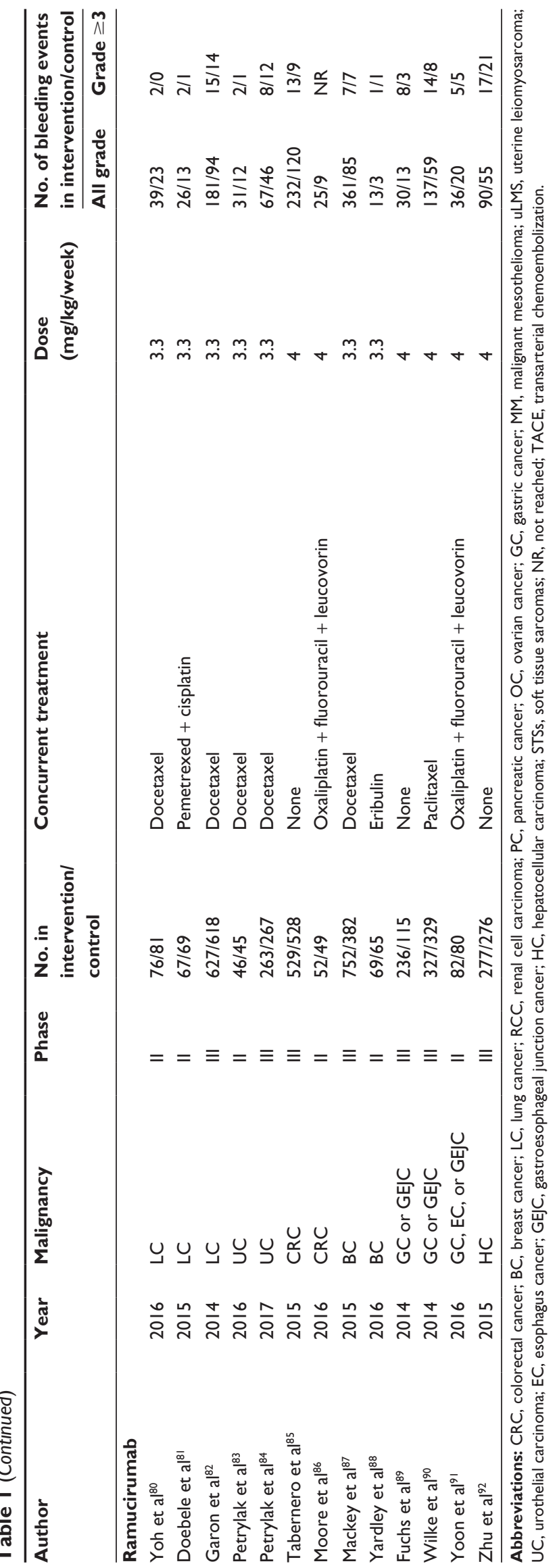

the risk of all-grade bleeding (RR: 1.94, 95\% CI: 1.76-2.13, $p<0.00001)$ and no difference was observed for the risk of high-grade bleeding (RR: 1.04, 95\% CI: $0.78-1.39, p=0.79$ ) compared with the control group. RR of all-grade and highgrade bleeding according to drug type is summarized in Tables 2 and 3, respectively.

In addition, we further assessed the risk of pulmonary hemorrhage of bevacizumab and ramucirumab in all lung cancer patients. The results showed that bevacizumab significantly increased the risk of all-grade (RR: $4.72,95 \% \mathrm{CI}$ : $1.99-11.19, p=0.0004)$ and high-grade pulmonary hemorrhage (RR: 3.97, 95\% CI: $1.70-9.29, p=0.001$ ), but no significant differences in the risk of all-grade (RR: 1.09, 95\% CI: $0.76-1.57, p=0.64)$ and high-grade (RR: $1.22,95 \% \mathrm{CI}$ : $0.35-4.21, p=0.75)$ pulmonary hemorrhage were observed for ramucirumab. RR of all-grade and high-grade pulmonary hemorrhage is shown in Figures 4 and 5, respectively.

\section{RR according to drug dosage}

In the subgroup analysis by dosage, the increased risk of allgrade and high-grade bleeding was observed in both low-dose and high-dose angiogenesis inhibitors.

The risks of all-grade bleeding were comparable between patients with low-dose angiogenesis inhibitors (RR: 2.46, 95\% CI: 1.95-3.11) and high-dose angiogenesis inhibitors (RR: 2.34, 95\% CI: 2.00-2.73) (Table 2). The risk of highgrade bleeding was more frequently observed in patients with high-dose angiogenesis inhibitors (RR: 2.17, 95\% CI: 1.79-2.64) than in those with low-dose angiogenesis inhibitors (RR: 1.31, 95\% CI: 1.06-1.60) (Table 3).

\section{RR according to tumor type}

Studies were further stratified according to tumor type (colorectal cancer vs non-colorectal tumors). Increased risk of all-grade and high-grade bleeding was observed in both the colorectal cancer arm and non-colorectal tumors arm. The risks of all-grade (RRs for colorectal cancer and non-colorectal tumors were 2.24, 95\% CI: 1.58-3.19 and 2.42, 95\% CI: 2.09-2.80, respectively) (Table 2) and high-grade bleeding (RRs for colorectal cancer and non-colorectal tumors were 1.52, 95\% CI: 1.13-2.03 and 1.77, 95\% CI: 1.50-2.09, respectively) (Table 3) were comparable between patients with colorectal cancer and non-colorectal tumors.

\section{Publication bias}

To minimize publication bias, we selected papers strictly according to the inclusion criteria. Furthermore, a funnel plot 


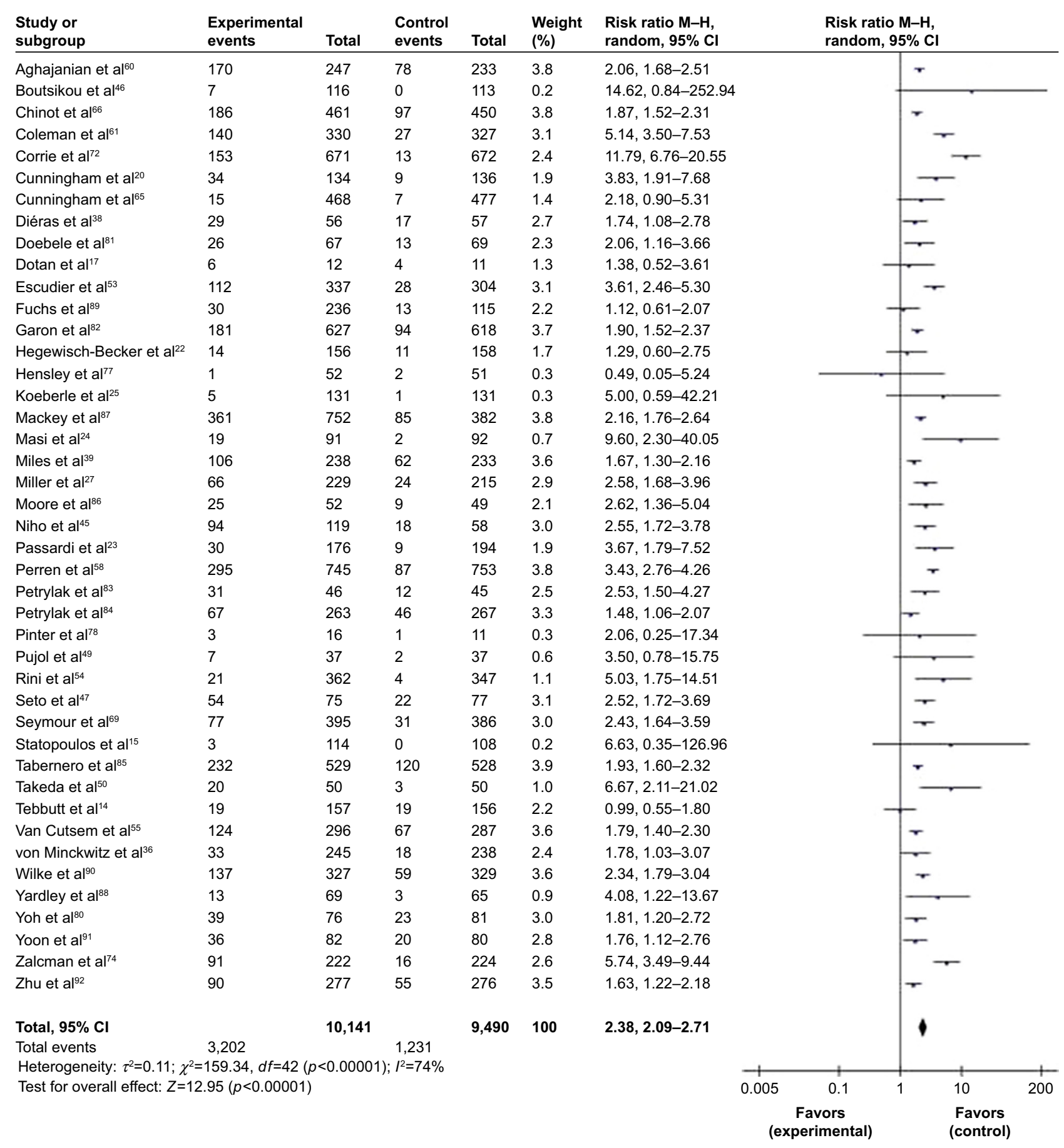

Figure 2 RR of all-grade bleeding.

Abbreviations: $\mathrm{M}-\mathrm{H}$, Mantel-Haenszel; RR, relative risk.

was used to detect publication bias and no apparent bias was found according to it for all-grade and high-grade bleeding.

\section{Discussion}

To the best of our knowledge, this is the first and the largest meta-analysis to assess the risk of bleeding associated with antiangiogenic monoclonal antibodies bevacizumab and ramucirumab. The results of our meta-analysis showed a significant 2.38-fold increased all-grade bleeding risk and a 1.71-fold increased high-grade bleeding risk with these agents. A similar risk of bleeding is also associated with other VEGF receptor tyrosine kinase inhibitors. ${ }^{93}$

In order to identify potential risk factors, we performed subgroup analysis according to drug types. The results 


\begin{tabular}{|c|c|c|c|c|c|c|c|c|}
\hline \multirow{2}{*}{$\begin{array}{l}\begin{array}{l}\text { Study or } \\
\text { subgroup }\end{array} \\
\text { Aghajanian et al }\left.\right|^{60}\end{array}$} & $\begin{array}{l}\text { Experimental } \\
\text { events }\end{array}$ & al ${ }_{\text {Total }}$ & $\begin{array}{l}\text { Control } \\
\text { events }\end{array}$ & Total & $\begin{array}{l}\text { Weight } \\
(\%)\end{array}$ & $\begin{array}{l}\text { Risk ratio } \mathrm{M}-\mathrm{H} \text {, } \\
\text { fixed, } 95 \% \mathrm{Cl}\end{array}$ & $\begin{array}{l}\text { Risk ra } \\
\text { fixed, } 9\end{array}$ & $\begin{array}{l}\text { tio } \mathrm{M}-\mathrm{H} \text {, } \\
5 \% \% \mathrm{Cl}\end{array}$ \\
\hline & 15 & 247 & 2 & 233 & 0.7 & $7.07,1.64-30.60$ & & $\ldots$ \\
\hline Allegra et al ${ }^{13}$ & 25 & 1,328 & 25 & 1,321 & 8.5 & $1.00,0.58-1.73$ & & - \\
\hline Balana et al ${ }^{68}$ & 5 & 48 & 0 & 45 & 0.2 & $10.33,0.59-181.54$ & & \\
\hline Bennouna et al $1^{19}$ & 5 & 401 & 1 & 409 & 0.3 & $8.16,1.06-64.94$ & & \\
\hline Boutsikou et al ${ }^{46}$ & 3 & 116 & 0 & 113 & 0.2 & $6.82,0.38-130.57$ & & \\
\hline Brufsky et al ${ }^{30}$ & 8 & 458 & 0 & 221 & 0.2 & $8.22,0.48-141.81$ & & \\
\hline Burger et al ${ }^{57}$ & 14 & 500 & 5 & 601 & 1.7 & $2.77,1.00-7.64$ & & $\cdots$ \\
\hline Cameron et al ${ }^{34}$ & 8 & 1,288 & 2 & 1,271 & 0.7 & $3.06,0.84-18.55$ & & \\
\hline Cao et $\mathrm{al}^{21}$ & 5 & 65 & 0 & 77 & 0.2 & $13.00,0.73-230.76$ & & \\
\hline Chinot et al ${ }^{16}$ & 15 & 451 & 5 & 450 & 2.7 & $1.83,0.78-4.27$ & & $\therefore$ \\
\hline Chisholm et al ${ }^{79}$ & 2 & 71 & 6 & 79 & 1.9 & $0.37,0.60-1.78$ & & I \\
\hline Coleman et al $\left.\right|^{61}$ & 6 & 330 & 3 & 327 & 1.0 & $1.98,0.50-7.86$ & & 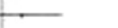 \\
\hline Corrie et $\mathrm{al}^{72}$ & 1 & 671 & 1 & 672 & 0.3 & $1.00,0.06-15.98$ & & \\
\hline Coudert et a $\left.\right|^{35}$ & 0 & 47 & 0 & 25 & & Not estimable & & \\
\hline Cunningham et al ${ }^{20}$ & 0 & 134 & 1 & 136 & 0.5 & $0.34,0.01-8.23$ & & \\
\hline Cunningham et al ${ }^{65}$ & 2 & 458 & 2 & 477 & 0.7 & $1.02,0.14-7.21$ & & \\
\hline De Gramont et al ${ }^{18}$ & 14 & 1,145 & 6 & 1,126 & 2.1 & $2.29,0.88-5.95$ & & 5 \\
\hline Diéras et al $\left.\right|^{38}$ & 0 & 58 & 0 & 57 & & Not estimable & & \\
\hline Doebele et a| $\left.\right|^{81}$ & 2 & 67 & 1 & 69 & 0.3 & $2.06,0.19-22.18$ & & \\
\hline Dotan et $\mathrm{al}^{17}$ & 0 & 12 & 0 & 11 & & Not estimable & & \\
\hline Escudier et al $\left.\right|^{53}$ & 11 & 337 & 1 & 304 & 0.4 & $9.32,1.20-78.41$ & & \\
\hline Fuchs et al $\left.\right|^{89}$ & 8 & 236 & 3 & 115 & 1.4 & $1.30,0.35-4.81$ & & - \\
\hline Garon et al ${ }^{82}$ & 15 & 627 & 14 & 618 & 4.8 & $1.06,0.51-2.17$ & & - \\
\hline Gianni et al ${ }^{33}$ & 3 & 215 & 1 & 206 & 0.3 & $2.47,0.30-27.41$ & & - \\
\hline Giantonio et al11 & 10 & 287 & 1 & 285 & 0.3 & $9.93,1.28-77.07$ & & \\
\hline Gilbert et $a^{67}$ & 4 & 250 & 2 & 233 & 0.7 & $1.79,0.33-9.70$ & & \\
\hline Guan et al ${ }^{16}$ & 1 & 141 & 1 & 70 & 0.3 & $0.50,0.00-7.82$ & & \\
\hline Hegewisch-Becker et al22 & 0 & 156 & 1 & 158 & 0.5 & $0.34,0.01-8.22$ & & \\
\hline Hensley et $\mathrm{al}^{77}$ & 0 & 52 & 1 & 51 & 0.5 & $0.33,0.01-7.85$ & & \\
\hline Herbst et a $\left.\right|^{42}$ & 3 & 39 & 1 & 42 & 0.3 & $3.23,0.35-29.77$ & & \\
\hline Herbst et al ${ }^{44}$ & 10 & 313 & 7 & 313 & 2.4 & $1.43,0.55-3.71$ & & - \\
\hline Hurwitz et a ${ }^{9}$ & 12 & 393 & 10 & 397 & 3.4 & $1.21,0.53-2.77$ & & - \\
\hline Johnson et a $\left.\right|^{40}$ & 6 & 65 & 0 & 32 & 0.2 & $6.40,0.37-110.26$ & & \\
\hline Kabbinavar et al ${ }^{8}$ & 3 & 67 & 0 & 35 & 0.2 & $3.71,0.20-69.79$ & & \\
\hline Kabbinavar et al ${ }^{10}$ & 5 & 100 & 3 & 104 & 1.0 & $1.73,0.43-7.06$ & & - \\
\hline Karayama et al ${ }^{51}$ & 0 & 46 & 0 & 36 & & Not estimable & & \\
\hline Kay et $\mathrm{al}^{70}$ & 1 & 33 & 0 & 22 & 0.2 & $2.91,0.12-04.95$ & & \\
\hline Kelly et a $7^{75}$ & 35 & 504 & 16 & 506 & 5.4 & $2.19,1.23-3.91$ & & 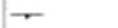 \\
\hline Kim et $\mathrm{al}^{71}$ & 2 & 143 & 5 & 69 & 2.3 & $0.19,0.04-0.97$ & & \\
\hline Kindler et al ${ }^{56}$ & 5 & 277 & 4 & 263 & 1.4 & $1.39,0.32-4.37$ & & $m$ \\
\hline Kindler et al ${ }^{13}$ & 4 & 53 & 1 & 55 & 0.3 & $4.15,0.48-35.05$ & & \\
\hline Koeberle et $\mathrm{al}^{25}$ & 0 & 131 & 0 & 131 & & Not estimable & & \\
\hline Mackey et al ${ }^{87}$ & 7 & 752 & 7 & 382 & 3.2 & $0.51,0.10-1.44$ & $\rightarrow$ & 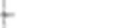 \\
\hline Masi et al ${ }^{24}$ & 0 & 91 & 0 & 92 & & Not estimable & & \\
\hline Miles et $a^{29}$ & 5 & 499 & 2 & 231 & 0.9 & $1.15,0.23-5.92$ & & \\
\hline Miles et $a^{139}$ & 2 & 238 & 2 & 233 & 0.7 & $0.98,0.14-6.89$ & & \\
\hline Miller et $\mathrm{al}^{27}$ & 1 & 229 & 1 & 215 & 0.4 & $0.94,0.06-14.92$ & & \\
\hline Miller et a ${ }^{28}$ & 2 & 355 & 0 & 346 & 0.2 & $4.74,0.23-98.32$ & 一 & \\
\hline Niho et al ${ }^{45}$ & 2 & 119 & 0 & 68 & 0.2 & $2.46,0.12-50.39$ & & \\
\hline Ohtsu et al $\left.\right|^{62}$ & 9 & 346 & 8 & 201 & 3.1 & $0.99,0.40-2.46$ & $=$ & t- \\
\hline Okines et al ${ }^{13}$ & 1 & 90 & 3 & 101 & 1.0 & $0.34,0.04-3.25$ & & — \\
\hline Perren et al ${ }^{58}$ & 9 & 746 & 2 & 753 & 0.7 & $4.56,0.99-20.96$ & & \\
\hline Petrylak et a $\left.\right|^{83}$ & 2 & 46 & 1 & 45 & 0.3 & $1.96,0.18-20.53$ & & $\rightarrow$ \\
\hline Petrylak et al ${ }^{84}$ & 8 & 263 & 12 & 267 & 4.0 & $0.66,0.28-1.63$ & - & 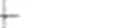 \\
\hline Pinter et $a^{l^{78}}$ & 3 & 16 & 1 & 11 & 0.4 & $2.06,0.25-17.34$ & & - \\
\hline Pujade-Lauraine et al $\left.\right|^{59}$ & 2 & 179 & 2 & 181 & 0.7 & $1.01,0.14-7.10$ & & - \\
\hline Pujol et al ${ }^{49}$ & 0 & 37 & 0 & 37 & & Not estimable & & \\
\hline Reck et al $\left.\right|^{43}$ & 28 & 650 & 5 & 327 & 2.7 & $2.32,0.97-5.54$ & & 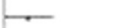 \\
\hline Rini et al ${ }^{54}$ & 4 & 352 & 1 & 347 & 0.3 & $3.83,0.43-34.14$ & & \\
\hline Robert et al ${ }^{31}$ & 14 & 617 & 1 & 400 & 0.5 & $6.91,0.91-52.33$ & & \\
\hline Saltz et al ${ }^{12}$ & 13 & 694 & 8 & 675 & 2.4 & $1.56,0.66-3.79$ & & - \\
\hline Sandler et a $\left.\right|^{41}$ & 19 & 427 & 3 & 440 & 1.0 & $6.53,1.95-25.89$ & & — \\
\hline Seto et al $\left.\right|^{47}$ & 2 & 75 & 0 & 77 & 0.2 & $0.13,0.25-105.14$ & & \\
\hline Seymour et al $\left.\right|^{69}$ & 5 & 395 & 1 & 388 & 0.3 & $7.02,0.01-62.21$ & & \\
\hline Shen et al ${ }^{164}$ & 12 & 100 & 4 & 101 & 1.4 & $3.03,1.01-0.08$ & & $\because$ \\
\hline Sikov et a $a^{37}$ & 7 & 215 & 0 & 218 & 0.2 & $15.21,0.07-254.64$ & & \\
\hline Snoeren et $a^{26}$ & 0 & 39 & 1 & 36 & 0.5 & $0.31,0.01-7.34$ & & {[} \\
\hline Tabernero et al ${ }^{85}$ & 13 & 529 & 9 & 528 & 3.1 & $1.44,0.62-3.34$ & & - \\
\hline Takeda et al ${ }^{50}$ & 0 & 50 & 0 & 60 & & Not estimable & & \\
\hline Tebbutt et al ${ }^{14}$ & 2 & 157 & 4 & 158 & 1.4 & $0.50,0.09-2.67$ & & - \\
\hline Tewari et al ${ }^{76}$ & 10 & 220 & 2 & 219 & 0.7 & $4.98,1.10-22.45$ & & \\
\hline Tiseo et a $a^{52}$ & 0 & 96 & 0 & 103 & & Not estimable & & \\
\hline Van Cutsem et al ${ }^{55}$ & 22 & 296 & 16 & 287 & 5.5 & $1.33,0.71-2.49$ & & - \\
\hline von Minckwitz et al $\left.\right|^{32}$ & 4 & 958 & 3 & 289 & 1.0 & $1.35,0.30-0.02$ & & \\
\hline von Minckwitz et a $\left.\right|^{36}$ & 1 & 246 & 4 & 238 & 1.4 & $0.24,0.03-2.16$ & & - \\
\hline Wilke et a $a^{90}$ & 14 & 327 & 8 & 329 & 2.7 & $1.76,0.76-4.14$ & & - \\
\hline Yardley et al ${ }^{88}$ & 1 & 59 & 1 & 65 & 0.3 & $0.94,0.06-14.75$ & & \\
\hline Yoh et al $\left.\right|^{80}$ & 2 & 76 & 0 & 81 & 0.2 & $5.32,0.26-109.15$ & & \\
\hline Yoon et a $\left.\right|^{91}$ & 5 & 82 & 5 & 80 & 1.7 & $0.98,0.29-3.24$ & — & - \\
\hline Zalcman et $\mathrm{al}^{74}$ & 2 & 222 & 0 & 224 & 0.2 & $5.04,0.24-104.49$ & & \\
\hline Zhou et a a ${ }^{48}$ & 2 & 140 & 1 & 134 & 0.3 & $1.91,0.18-20.87$ & & \\
\hline Zhu et al ${ }^{92}$ & 17 & 277 & 21 & 276 & 7.1 & $0.81,0.44-1.50$ & & \\
\hline Total, $95 \% \mathrm{Cl}$ & & 24,062 & & 21,855 & 100 & $1.71,1.48-1.97$ & & 1 \\
\hline Total events & 626 & & 276 & & & & & \\
\hline Heterogeneity: $\chi^{2}=90.19, a$ & $72(p=0.07) ; 1^{2}=$ & $=20 \%$ & & & & & & \\
\hline Test for overall effect: $Z=7$. & $(p<0.00001)$ & & & & & & $\begin{array}{l}.1 \\
\text { ors } \\
\text { nental) }\end{array}$ & $\begin{array}{l}\text { Favors } \\
\text { (control }\end{array}$ \\
\hline
\end{tabular}

Figure 3 RR of high-grade bleeding.

Abbreviations: $\mathrm{M}-\mathrm{H}$, Mantel-Haenszel; RR, relative risk.

showed that ramucirumab differed from bevacizumab in terms of the risk of high-grade bleeding and the risk of all-grade and high-grade pulmonary hemorrhage in lung cancer patients. The mechanisms underlying these differences remained unclear. A possible explanation was that bevacizumab, as an anti-VEGF-A agent, specified both VEGFR-1 and VEGFR-2, whereas ramucirumab was only specified for VEGFR-2. VEGFR-2 was the major mediator 
Table 2 RR of all-grade bleeding associated with angiogenesis inhibitors in the subgroup analysis

\begin{tabular}{|c|c|c|c|c|}
\hline \multirow[t]{2}{*}{ Bleeding } & \multirow{2}{*}{$\begin{array}{l}\text { No. of } \\
\text { trials }\end{array}$} & \multicolumn{2}{|c|}{ No. of events/total (\%) } & \multirow[t]{2}{*}{ RR, 95\% Cl } \\
\hline & & Treatment & Control & \\
\hline \multicolumn{5}{|l|}{ Type of drug } \\
\hline Bevacizumab & 30 & I,934/6,738 (28.7) & $679 / 6,586(10.3)$ & $2.73,2.24-3.33$ \\
\hline Ramucirumab & 13 & I,268/3,403 (37.3) & $552 / 2,904(19.0)$ & $1.94,1.76-2.13$ \\
\hline \multicolumn{5}{|l|}{ Drug dosage } \\
\hline Low dose & 22 & I,452/5,220 (27.8) & $508 / 4,863(10.4)$ & $2.46,1.95-3.11$ \\
\hline High dose & 21 & I,750/4,92। (35.6) & $723 / 4,627(15.6)$ & $2.34,2.00-2.73$ \\
\hline \multicolumn{5}{|l|}{ Tumor types } \\
\hline Colorectal cancer & 10 & $387 / I, 552(24.9)$ & I84/I,563 (II.8) & $2.24,1.58-3.19$ \\
\hline Non-colorectal cancer & 33 & 2,815/8,589 (32.8) & $\mathrm{I}, 047 / 7,927$ (I 3.2) & $2.42,2.09-2.80$ \\
\hline
\end{tabular}

Abbreviation: $R R$, relative risk.

of VEGF-driven responses in endothelial cells. The precise function of VEGFR-1 was not entirely established and some studies showed that VEGFR-1 could also regulate proliferation and survival of endothelial cells. ${ }^{94-97}$ Increased level of tumor VEGFR-1 expression has been shown to be associated with high tumor angiogenesis. ${ }^{96}$ VEGF/VEGFR-1 signalingmediated tumor cell monocyte chemoattractant protein-1 expression could represent a mechanism responsible for the tumor angiogenic switch. ${ }^{97}$ Therefore, bevacizumab increased the risk of bleeding by inhibiting both VEGFR-1 and VEGFR-2. Squamous cell tumors are more frequently centrally located and have a greater tendency to cavitate as compared to adenocarcinoma, which is the main risk factor of pulmonary hemorrhage. ${ }^{98}$ The difference in the risk of pulmonary hemorrhage caused bevacizumab to be used only for non-squamous NSCLC and ramucirumab to be used for any tumor histology of NSCLC.

Our study also demonstrated that both low-dose and high-dose angiogenesis inhibitors increased the risk of bleeding. The risk of high-grade bleeding was more frequently observed in patients with high-dose angiogenesis inhibitors, suggesting that the risk may be dose-dependent and close supervision and careful management should be emphasized especially in patients with high dosage.

In a meta-analysis of bevacizumab, patients with colorectal cancer were found to have the highest risk of bleeding compared to other tumors. ${ }^{99}$ For colorectal cancer patients, high-grade bleeding such as perforation was commonly fatal and life threatening. ${ }^{100}$ Therefore, we performed a subgroup analysis according to colorectal cancer and non-colorectal tumors in order to identify the potential risk factors. Results showed that the risk of all-grade and high-grade bleeding was comparable between patients with colorectal cancer and non-colorectal tumors, suggesting that the increased risk of bleeding is associated with many tumor types.

\section{Limitations}

There are several limitations in this meta-analysis. First, we performed stratification analysis only for colorectal cancer and non-colorectal tumor types because too many tumor types were included in the analysis and assessment was difficult. Second, we did not evaluate the risk of pulmonary hemorrhage between bevacizumab and ramucirumab in

Table 3 RR of high-grade bleeding associated with angiogenesis inhibitors in the subgroup analysis

\begin{tabular}{|c|c|c|c|c|}
\hline \multirow[t]{2}{*}{ Bleeding } & \multirow{2}{*}{$\begin{array}{l}\text { No. of } \\
\text { trials }\end{array}$} & \multicolumn{2}{|c|}{ No. of events/total (\%) } & \multirow[t]{2}{*}{ RR, 95\% Cl } \\
\hline & & Treatment & Control & \\
\hline \multicolumn{5}{|l|}{ Type of drug } \\
\hline Bevacizumab & 70 & $432 / 20,731(2.1)$ & $194 / 19,000(1.0)$ & $1.98,1.68-2.34$ \\
\hline Ramucirumab & 12 & $94 / 3,351(2.8)$ & $82 / 2,855(2.9)$ & $1.04,0.78-1.39$ \\
\hline \multicolumn{5}{|l|}{ Drug dosage } \\
\hline Low dose & 37 & $203 / 10,569(1.9)$ & 149/10,089 (1.5) & $1.31,1.06-1.60$ \\
\hline High dose & 49 & $323 / 13,5 \mid 3(2.4)$ & $|35 /| 2,39 \mid(1.1)$ & $2.17,1.79-2.64$ \\
\hline \multicolumn{5}{|l|}{ Tumor types } \\
\hline Colorectal cancer & 18 & II I/5,868 (I.9) & $7 \mathrm{I} / 5,747$ (I.2) & $1.52,1.13-2.03$ \\
\hline Non-colorectal cancer & 64 & $4|5 /| 8,2 \mid 4(2.3)$ & $205 / 16,108(1.3)$ & $1.77,1.50-2.09$ \\
\hline
\end{tabular}

Abbreviation: RR, relative risk. 


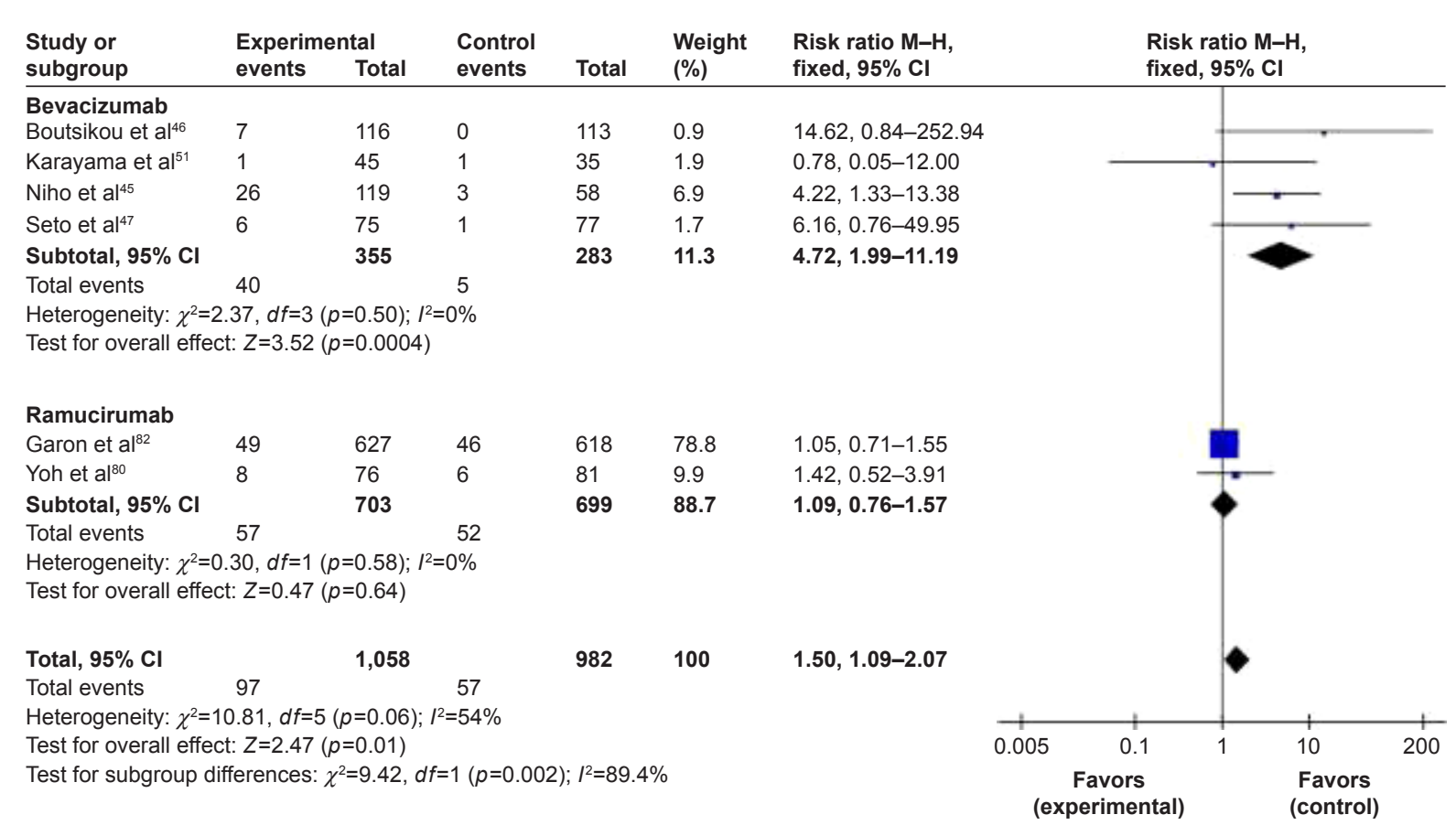

Figure 4 RR of all-grade pulmonary hemorrhage.

Abbreviations: $\mathrm{M}-\mathrm{H}$, Mantel-Haenszel; RR, relative risk.

\begin{tabular}{|c|c|c|c|c|c|c|c|c|c|}
\hline \multirow{2}{*}{$\begin{array}{l}\text { Study or } \\
\text { subgroup } \\
\text { Bevacizumab }\end{array}$} & \multicolumn{2}{|c|}{ Experimental } & \multirow[t]{2}{*}{$\begin{array}{l}\text { Control } \\
\text { events }\end{array}$} & \multirow[t]{2}{*}{ Total } & \multirow[t]{2}{*}{$\begin{array}{l}\text { Weight } \\
(\%)\end{array}$} & \multirow[t]{2}{*}{$\begin{array}{l}\text { Risk ratio } \mathrm{M}-\mathrm{H} \text {, } \\
\text { fixed, } 95 \% \mathrm{Cl}\end{array}$} & \multicolumn{3}{|c|}{$\begin{array}{l}\text { Risk ratio } \mathrm{M}-\mathrm{H} \text {, } \\
\text { fixed, } 95 \% \mathrm{Cl}\end{array}$} \\
\hline & & & & & & & & & \\
\hline Boutsikou et al ${ }^{46}$ & 3 & 116 & 0 & 113 & 4.4 & $6.82,0.36-130.57$ & & & \\
\hline Herbst et $\mathrm{al}^{42}$ & 2 & 39 & 0 & 42 & 4.2 & $5.38,0.27-108.58$ & & & \\
\hline Herbst et $a^{44}$ & 3 & 313 & 1 & 313 & 8.7 & $3.00,0.31-28.68$ & & & \\
\hline Johnson et $\mathrm{al}^{40}$ & 6 & 66 & 0 & 32 & 5.8 & $6.40,0.37-110.26$ & & & \\
\hline Karayama et al ${ }^{51}$ & 0 & 45 & 0 & 35 & & Not estimable & & & \\
\hline Niho et $a^{45}$ & 1 & 119 & 0 & 58 & 5.8 & $1.48,0.06-35.66$ & & & \\
\hline Pujol et al ${ }^{49}$ & 0 & 37 & 0 & 37 & & Not estimable & & & \\
\hline Reck et al ${ }^{43}$ & 8 & 659 & 2 & 327 & 23.2 & $1.98,0.42-9.29$ & & & \\
\hline Sandler et al ${ }^{41}$ & 8 & 427 & 1 & 440 & 8.6 & $8.24,1.04-65.63$ & & & \\
\hline Seto et $\mathrm{al}^{47}$ & 0 & 75 & 0 & 77 & & Not estimable & & & \\
\hline Takeda et al ${ }^{50}$ & 0 & 50 & 0 & 50 & & Not estimable & & & \\
\hline Tiseo et $\mathrm{al}^{52}$ & 0 & 95 & 0 & 103 & & Not estimable & & & \\
\hline Zhou et $\mathrm{al}^{48}$ & 0 & 140 & 0 & 134 & & Not estimable & & & \\
\hline Subtotal, $95 \% \mathrm{Cl}$ & & 2,181 & & 1,761 & 60.8 & $3.97,1.70-9.29$ & & & \\
\hline Total events & 31 & & 4 & & & & & & \\
\hline \multicolumn{10}{|c|}{ Heterogeneity: $\chi^{2}=1.96, d f=6(p=0.92) ; I^{2}=0 \%$} \\
\hline \multicolumn{10}{|c|}{ Test for overall effect: $Z=3.18(p=0.001)$} \\
\hline \multicolumn{10}{|l|}{ Ramucirumab } \\
\hline Garon et $\mathrm{al}^{82}$ & 4 & 627 & 4 & 618 & 35.0 & $0.99,0.25-3.92$ & & & \\
\hline Yoh et $\mathrm{al}^{80}$ & 1 & 76 & 0 & 81 & 4.2 & $3.19,0.13-77.25$ & & & \\
\hline Subtotal, $95 \% \mathrm{Cl}$ & & 703 & & 699 & 39.2 & $1.22,0.35-4.21$ & & & \\
\hline Total events & 5 & & 4 & & & & & & \\
\hline \multicolumn{10}{|c|}{ Heterogeneity: $\chi^{2}=0.44, d f=1(p=0.51) ; I^{2}=0 \%$} \\
\hline \multicolumn{10}{|c|}{ Test for overall effect: $Z=0.32(p=0.75)$} \\
\hline Total, $95 \% \mathrm{Cl}$ & & 2,884 & & 2,460 & 100 & $2.89,1.46-5.72$ & & & \\
\hline Total events & 36 & & 8 & & & & & & \\
\hline \multicolumn{10}{|c|}{ Heterogeneity: $\chi^{2}=4.50, d f=8(p=0.81) ; I^{2}=0 \%$} \\
\hline Test for overall effe & $Z=3.05(p$ & $0.002)$ & & & & & 0.005 & 10 & 200 \\
\hline \multicolumn{7}{|c|}{ Test for subgroub differences: $\chi^{2}=2.37, d f=1(p=0.12) ; I^{2}=57.7 \%$} & $\begin{array}{c}\text { Favors } \\
\text { (experimental) }\end{array}$ & $\begin{array}{l}\text { Favors } \\
\text { (control) }\end{array}$ & \\
\hline
\end{tabular}

Figure 5 RR of high-grade pulmonary hemorrhage.

Abbreviations: $\mathrm{M}-\mathrm{H}$, Mantel-Haenszel; RR, relative risk. 
lung squamous cell carcinoma patients due to the small sample size or absence of original data. Finally, our literature search was limited to articles published in English leading to some selection bias.

\section{Conclusion}

Despite the limitations of our meta-analysis, we conclude that antiangiogenic monoclonal antibodies are associated with a significant increase in the risk of all-grade and high-grade bleeding. Ramucirumab may be different from bevacizumab in terms of the risk of high-grade bleeding and the risk of all-grade and high-grade pulmonary hemorrhage in lung cancer patients. Clinicians should be aware of this adverse effect and ensure close monitoring, especially in patients at high risk.

\section{Acknowledgment}

This study was supported by the Beijing Natural Science Foundation (7142125).

\section{Disclosure}

The authors report no conflicts of interest in this work.

\section{References}

1. Kamba T, McDonald DM. Mechanisms of adverse effects of anti-VEGF therapy for cancer. Br J Cancer. 2007;96:1788-1795.

2. Kerbel RS. Tumor angiogenesis. N Engl J Med. 2008;358: 2039-2049.

3. Hicklin DJ, Ellis LM. Role of the vascular endothelial growth factor pathway in tumor growth and angiogenesis. J Clin Oncol. 2005;23: 1011-1027.

4. Poole RM, Vaidya A. Ramucirumab: first global approval. Drugs. 2014;74:1047-1058.

5. Kilickap S, Abali H, Celik I. Bevacizumab, bleeding,thrombosis, and warfarin. J Clin Oncol. 2003;21:3542.

6. Moher D, Liberati A, Tetzlaff J, Altman DG; PRISMA Group. Preferred reporting items for systematic reviews and meta-analyses: the PRISMA statement. Ann Intern Med. 2009;151:264-269.

7. Higgins JP, Thompson SG, Deeks JJ, Altman DG. Measuring inconsistency in meta-analyses. BMJ. 2003;327:557-560.

8. Kabbinavar F, Hurwitz HI, Fehrenbacher L, et al. Phase II, randomized trial comparing bevacizumab plus fluorouracil (FU)/leucovorin (LV) with $\mathrm{FU} / \mathrm{LV}$ alone in patients with metastatic colorectal cancer. $J$ Clin Oncol. 2003;21:60-65.

9. Hurwitz H, Fehrenbacher L, Novotny W, et al. Bevacizumab plus irinotecan, fluorouracil, and leucovorin for metastatic colorectal cancer. N Engl J Med. 2004;350:2335-2342.

10. Kabbinavar FF, Schulz J, McCleod M, et al. Addition of bevacizumab to bolus fluorouracil and leucovorin in first-line metastatic colorectal cancer: results of a randomized phase II trial. J Clin Oncol. 2005;23: 3697-3705.

11. Giantonio BJ, Catalano PJ, Meropol NJ, et al. Bevacizumab in combination with oxaliplatin, fluorouracil, and leucovorin (FOLFOX4) for previously treated metastatic colorectal cancer: results from the Eastern Cooperative Oncology Group Study E3200. J Clin Oncol. 2007;25: 1539-1544.
12. Saltz LB, Clarke S, Diaz-Rubio E, et al. Bevacizumab in combination with oxaliplatin-based chemotherapy as first-line therapy in metastatic colorectal cancer: a randomized phase III study. J Clin Oncol. 2008; 26:2013-2019.

13. Allegra CJ, Yothers G, O'Connell MJ, et al. Initial safety report of NSABP C-08: a randomized phase III study of modified FOLFOX6 with or without bevacizumab for the adjuvant treatment of patients with stage II or III colon cancer. J Clin Oncol. 2009;27:3385-3390.

14. Tebbutt NC, Wilson K, Gebski VJ, et al. Capecitabine, bevacizumab, and mitomycin in first-line treatment of metastatic colorectal cancer: results of the Australasian Gastrointestinal Trials Group randomized phase III MAX study. J Clin Oncol. 2010;28:3191-3198.

15. Stathopoulos GP, Batziou C, Trafalis D, et al. Treatment of colorectal cancer with and without bevacizumab: a phase III study. Oncology. 2010;78:376-381.

16. Guan ZZ, Xu JM, Luo RC, et al. Efficacy and safety of bevacizumab plus chemotherapy in Chinese patients with metastatic colorectal cancer: a randomized phase III ARTIST trial. Chin J Cancer. 2011;30: 682-689.

17. Dotan E, Meropol NJ, Burtness B, et al. A phase II study of capecitabine, oxaliplatin, and cetuximab with or without bevacizumab as frontline therapy for metastatic colorectal cancer. A Fox Chase extramural research study. J Gastrointest Cancer. 2012;43:562-569.

18. de Gramont, Van Cutsem E, Schmoll HJ, et al. Bevacizumab plus oxaliplatin-based chemotherapy as adjuvant treatment for colon cancer (AVANT): a phase 3 randomised controlled trial. Lancet Oncol. 2012; 13:1225-1233.

19. Bennouna J, Sastre J, Arnold D, et al. Continuation of bevacizumab after first progression in metastatic colorectal cancer (ML18147): a randomised phase 3 trial. Lancet Oncol. 2013;14:29-37.

20. Cunningham D, Lang I, Marcuello E, et al. Bevacizumab plus capecitabine versus capecitabine alone in elderly patients with previously untreated metastatic colorectal cancer (AVEX): an open-label, randomised phase 3 trial. Lancet Oncol. 2013;14:1077-1085.

21. Cao R, Zhang S, Ma D, Hu L. A multi-center randomized phase II clinical study of bevacizumab plus irinotecan, 5-fluorouracil, and leucovorin (FOLFIRI) compared with FOLFIRI alone as second-line treatment for Chinese patients with metastatic colorectal cancer. Med Oncol. 2015;32:325-329.

22. Hegewisch-Becker S, Graeven U, Lerchenmuller CA, et al. Maintenance strategies after first-line oxaliplatin plus fluoropyrimidine plus bevacizumab for patients with metastatic colorectal cancer (AIO 0207): a randomised, non-inferiority, open-label, phase 3 trial. Lancet Oncol. 2015;16:1355-1369.

23. Passardi A, Nanni O, Tassinari D, et al. Effectiveness of bevacizumab added to standard chemotherapy in metastatic colorectal cancer: final results for first-line treatment from the ITACa randomized clinical trial. Ann Oncol. 2015;26:1201-1207.

24. Masi G, Salvatore L, Boni L, et al. Continuation or reintroduction of bevacizumab beyond progression to first-line therapy in metastatic colorectal cancer: final results of the randomized BEBYP trial. Ann Oncol. 2015;26:724-730.

25. Koeberle D, Betticher DC, von Moos R, et al. Bevacizumab continuation versus no continuation after first-line chemotherapy plus bevacizumab in patients with metastatic colorectal cancer: a randomized phase III non-inferiority trial (SAKK 41/06). Ann Oncol. 2015;26:709-714.

26. Snoeren N, van Hillegersberg R, Schouten SB, et al. Randomized phase III study to assess efficacy and safety of adjuvant CAPOX with or without bevacizumab in patients after resection of colorectal liver metastases: HEPATICA study. Neoplasia. 2017;19:93-99.

27. Miller KD, Chap LI, Holmes FA, et al. Randomized phase III trial of capecitabine compared with bevacizumab plus capecitabine in patients with previously treated metastatic breast cancer. J Clin Oncol. 2005; 23:792-799.

28. Miller K, Wang M, Gralow J, et al. Paclitaxel plus bevacizumab versus paclitaxel alone for metastatic breast cancer. $N$ Engl J Med. 2007; 357:2666-2676. 
29. Miles DW, Chan A, Dirix LY, et al. Phase III study of bevacizumab plus docetaxel compared with placebo plus docetaxel for the first-line treatment of human epidermal growth factor receptor 2-negative metastatic breast cancer. J Clin Oncol. 2010;28:3239-3247.

30. Brufsky AM, Hurvitz S, Perez E, et al. RIBBON-2: a randomized, double-blind, placebo-controlled, phase III trial evaluating the efficacy and safety of bevacizumab in combination with chemotherapy for second-line treatment of human epidermal growth factor receptor 2-negative metastatic breast cancer. J Clin Oncol. 2011;29: 4286-4293.

31. Robert NJ, Dieras V, Glaspy J, et al. RIBBON-1: randomized, doubleblind, placebo-controlled, phase III trial of chemotherapy with or without bevacizumab for first-line treatment of human epidermal growth factor receptor 2-negative, locally recurrent or metastatic breast cancer. J Clin Oncol. 2011;29:1252-1260.

32. von Minckwitz G, Eidtmann H, Rezai M, et al. Neoadjuvant chemotherapy and bevacizumab for HER2-negative breast cancer. $N$ Engl $J$ Med. 2012;366:299-309.

33. Gianni L, Romieu GH, Lichinitser M, et al. AVEREL: a randomized phase III trial evaluating bevacizumab in combination with docetaxel and trastuzumab as first-line therapy for HER2-positive locally recurrent/metastatic breast cancer. J Clin Oncol. 2013;31:1719-1725.

34. Cameron D, Brown J, Dent R, et al. Adjuvant bevacizumab-containing therapy in triple-negative breast cancer (BEATRICE): primary results of a randomised, phase 3 trial. Lancet Oncol. 2013;14:933-942.

35. Coudert B, Pierga JY, Mouret-Reynier MA, et al. Use of [(18)F]-FDG PET to predict response to neoadjuvant trastuzumab and docetaxel in patients with HER2-positive breast cancer, and addition of bevacizumab to neoadjuvant trastuzumab and docetaxel in [(18)F]-FDG PETpredicted non-responders (AVATAXHER): an open-label, randomised phase 2 trial. Lancet Oncol. 2014;15:1493-1502.

36. von Minckwitz G, Puglisi F, Cortes J, et al. Bevacizumab plus chemotherapy versus chemotherapy alone as second-line treatment for patients with HER2-negative locally recurrent or metastatic breast cancer after first-line treatment with bevacizumab plus chemotherapy (TANIA): an open-label, randomised phase 3 trial. Lancet Oncol. 2014;15: $1269-1278$

37. Sikov WM, Berry DA, Perou CM, et al. Impact of the addition of carboplatin and/or bevacizumab to neoadjuvant once-per-week paclitaxel followed by dose-dense doxorubicin and cyclophosphamide on pathologic complete response rates in stage II to III triple-negative breast cancer: CALGB 40603 (Alliance). J Clin Oncol. 2015;33:13-21.

38. Diéras V, Wildiers H, Jassem J, et al. Trebananib (AMG 386) plus weekly paclitaxel with or without bevacizumab as first-line therapy for HER2-negative locally recurrent or metastatic breast cancer: a phase 2 randomized study. Breast. 2015;24:182-190.

39. Miles D, Cameron D, Bondarenko I, et al. Bevacizumab plus paclitaxel versus placebo plus paclitaxel as first-line therapy for HER2negative metastatic breast cancer (MERiDiAN): a double-blind placebo-controlled randomised phase III trial with prospective biomarker evaluation. Eur J Cancer. 2017;70:146-155.

40. Johnson DH, Fehrenbacher L, Novotny WF, et al. Randomized phase II trial comparing bevacizumab plus carboplatin and paclitaxel with carboplatin and paclitaxel alone in previously untreated locally advanced or metastatic non-small-cell lung cancer. J Clin Oncol. 2004;22:2184-2191

41. Sandler A, Gray R, Perry MC, et al. Paclitaxel-carboplatin alone or with bevacizumab for non-small-cell lung cancer. N Engl J Med. 2006; 355:2542-2550.

42. Herbst RS, O'Neill VJ, Fehrenbacher L, et al. Phase II study of efficacy and safety of bevacizumab in combination with chemotherapy or erlotinib compared with chemotherapy alone for treatment of recurrent or refractory non small-cell lung cancer. J Clin Oncol. 2007;25: 4743-4750.

43. Reck M, von Pawel J, Zatloukal P, et al. Phase III trial of cisplatin plus gemcitabine with either placebo or bevacizumab as first-line therapy for nonsquamous non-small-cell lung cancer: AVAil. J Clin Oncol. 2009;27:1227-1234.
44. Herbst RS, Ansari R, Bustin F, et al. Efficacy of bevacizumab plus erlotinib versus erlotinib alone in advanced non-small-cell lung cancer after failure of standard first-line chemotherapy (BeTa): a double-blind, placebo-controlled, phase 3 trial. Lancet. 2011;377:1846-1854.

45. Niho S, Kunitoh H, Nokihara H, et al. Randomized phase II study of first-line carboplatin-paclitaxel with or without bevacizumab in Japanese patients with advanced non-squamous non-small-cell lung cancer. Lung Cancer. 2012;76:362-367.

46. Boutsikou E, Kontakiotis T, Zarogoulidis P, et al. Docetaxel-carboplatin in combination with erlotinib and/or bevacizumab in patients with non-small cell lung cancer. Onco Targets Ther. 2013;6:125-134.

47. Seto T, Kato T, Nishio M, et al. Erlotinib alone or with bevacizumab as first-line therapy in patients with advanced non-squamous non-smallcell lung cancer harbouring EGFR mutations (JO25567): an openlabel, randomised, multicentre, phase 2 study. Lancet Oncol. 2014;15: $1236-1244$.

48. Zhou C, Wu YL, Chen G, et al. BEYOND: a randomized, double-blind, placebo-controlled, multicenter, phase III study of first-line carboplatin/ paclitaxel plus bevacizumab or placebo in Chinese patients with advanced or recurrent nonsquamous non-small-cell lung cancer. J Clin Oncol. 2015;33:2197-2204

49. Pujol JL, Lavole A, Quoix E, et al. Randomized phase II-III study of bevacizumab in combination with chemotherapy in previously untreated extensive small-cell lung cancer: results from the IFCT-0802 trial. Ann Oncol. 2015;26:908-914.

50. Takeda M, Yamanaka T, Seto T, et al. Bevacizumab beyond disease progression after first-line treatment with bevacizumab plus chemotherapy in advanced nonsquamous non-small cell lung cancer (West Japan Oncology Group 5910L): an open-label, randomized, phase 2 trial. Cancer. 2016;122:1050-1059.

51. Karayama M, Inui N, Fujisawa T, et al. Maintenance therapy with pemetrexed and bevacizumab versus pemetrexed monotherapy after induction therapy with carboplatin, pemetrexed, and bevacizumab in patients with advanced non-squamous non small cell lung cancer. Eur J Cancer. 2016;58:30-37.

52. Tiseo M, Boni L, Ambrosio F, et al. Italian, multicenter, phase III, randomized study of cisplatin plus etoposide with or without bevacizumab as first-line treatment in extensive-disease small-cell lung cancer: the GOIRC-AIFA FARM6PMFJM trial.J Clin Oncol. 2017;35: 1281-1287.

53. Escudier B, Pluzanska A, Koralewski P, et al. Bevacizumab plus interferon alfa-2a for treatment of metastatic renal cell carcinoma: a randomised, double-blind phase III trial. Lancet. 2007;370:2103-2111.

54. Rini BI, Halabi S, Rosenberg JE, et al. Phase III trial of bevacizumab plus interferon alfa versus interferon alfa monotherapy in patients with metastatic renal cell carcinoma: final results of CALGB 90206. J Clin Oncol. 2010;28:2137-2143.

55. Van Cutsem E, Vervenne WL, Bennouna J, et al. Phase III trial of bevacizumab in combination with gemcitabine and erlotinib in patients with metastatic pancreatic cancer. J Clin Oncol. 2009;27:2231-2237.

56. Kindler HL, Niedzwiecki D, Hollis D, et al. Gemcitabine plus bevacizumab compared with gemcitabine plus placebo in patients with advanced pancreatic cancer: phase III trial of the Cancer and Leukemia Group B (CALGB 80303). J Clin Oncol. 2010;28:3617-3622.

57. Burger RA, Brady MF, Bookman MA, et al. Incorporation of bevacizumab in the primary treatment of ovarian cancer. NEngl J Med. 2011; 365:2473-2483.

58. Perren TJ, Swart AM, Pfisterer J, et al. A phase 3 trial of bevacizumab in ovarian cancer. N Engl J Med. 2011;365:2484-2496.

59. Pujade-Lauraine E, Hilpert F, Weber B, et al. Bevacizumab combined with chemotherapy for platinum-resistant recurrent ovarian cancer: the AURELIA open-label randomized phase III trial. J Clin Oncol. 2014; 32:1302-1308.

60. Aghajanian C, Goff B, Nycum LR, Wang YV, Husain A, Blank SV. Final overall survival and safety analysis of OCEANS, a phase 3 trial of chemotherapy with or without bevacizumab in patients with platinum-sensitive recurrent ovarian cancer. Gynecol Oncol. 2015;139: $10-16$. 
61. Coleman RL, Brady MF, Herzog TJ, et al. Bevacizumab and paclitaxelcarboplatin chemotherapy and secondary cytoreduction in recurrent, platinum-sensitive ovarian cancer (NRG Oncology/Gynecologic Oncology Group study GOG-0213): a multicentre, open-label, randomised, phase 3 trial. Lancet Oncol. 2017;18:779-791.

62. Ohtsu A, Shah MA, Van Cutsem E, et al. Bevacizumab in combination with chemotherapy as first-line therapy in advanced gastric cancer: a randomized, double-blind, placebo-controlled phase III study. J Clin Oncol. 2011;29:3968-3976.

63. Okines AF, Langley RE, Thompson LC, et al. Bevacizumab with perioperative epirubicin, cisplatin and capecitabine (ECX) in localised gastro-oesophageal adenocarcinoma: a safety report. Ann Oncol. 2013; 24:702-709.

64. Shen L, Li J, Xu J, et al. Bevacizumab plus capecitabine and cisplatin in Chinese patients with inoperable locally advanced or metastatic gastric or gastroesophageal junction cancer: randomized, double-blind, phase III study (AVATAR study). Gastric Cancer. 2015;18:168-176.

65. Cunningham D, Stenning SP, Smyth EC, et al. Peri-operative chemotherapy with or without bevacizumab in operable oesophagogastric adenocarcinoma (UK Medical Research Council ST03): primary analysis results of a multicentre, open-label, randomised phase 2-3 trial. Lancet Oncol. 2017;18:357-370.

66. Chinot OL, Wick W, Mason W, et al. Bevacizumab plus radiotherapytemozolomide for newly diagnosed glioblastoma. $N$ Engl J Med. 2014;370:709-722.

67. Gilbert MR, Dignam JJ, Armstrong TS, et al. A randomized trial of bevacizumab for newly diagnosed glioblastoma. $N$ Engl J Med. 2014; 370:699-708.

68. Balana C, De Las Penas R, Sepúlveda JM, et al. Bevacizumab and temozolomide versus temozolomide alone as neoadjuvant treatment in unresected glioblastoma: the GENOM 009 randomized phase II trial. J Neurooncol. 2016;127:569-579.

69. Seymour JF, Pfreundschuh M, Trneny M, et al. R-CHOP with or without bevacizumab in patients with previously untreated diffuse large B-cell lymphoma: final MAIN study outcomes. Haematologica. 2014; 99:1343-1349.

70. Kay NE, Strati P, LaPlant BR, et al. A randomized phase II trial comparing chemoimmunotherapy with or without bevacizumab in previously untreated patients with chronic lymphocytic leukemia. Oncotarget. 2016;48:78269-78280.

71. Kim KB, Sosman JA, Fruehauf JP, et al. BEAM: a randomized phase II study evaluating the activity of bevacizumab in combination with carboplatin plus paclitaxel in patients with previously untreated advanced melanoma. J Clin Oncol. 2012;30:34-41.

72. Corrie PG, Marshall A, Dunn JA, et al. Adjuvant bevacizumab in patients with melanoma at high risk of recurrence (AVAST-M): preplanned interim results from a multicentre, open-label, randomised controlled phase 3 study. Lancet Oncol. 2014;15:620-630.

73. Kindler HL, Karrison TG, Gandara DR, et al. Multicenter, double-blind, placebo-controlled, randomized phase II trial of gemcitabine/cisplatin plus bevacizumab or placebo in patients with malignant mesothelioma. J Clin Oncol. 2012;30:2509-2515.

74. Zalcman G, Mazieres J, Margery J, et al. Bevacizumab for newly diagnosed pleural mesothelioma in the Mesothelioma Avastin Cisplatin Pemetrexed Study (MAPS): a randomised, controlled, open-label, phase 3 trial. Lancet. 2016;387:1405-1414.

75. Kelly WK, Halabi S, Carducci M, et al. Randomized, double-blind, placebo-controlled phase III trial comparing docetaxel and prednisone with or without bevacizumab in men with metastatic castrationresistant prostate cancer: CALGB 90401. J Clin Oncol. 2012;30: $1534-1540$.

76. Tewari KS, Sill MW, Long HJ, et al. Improved survival with bevacizumab in advanced cervical cancer. $N$ Engl J Med. 2014;370:734-743.

77. Hensley ML, Miller A, O’Malley DM, et al. Randomized phase III trial of gemcitabine plus docetaxel plus bevacizumab or placebo as first-line treatment for metastatic uterine leiomyosarcoma: an NRG Oncology/Gynecologic Oncology Group study. J Clin Oncol. 2015;33: $1180-1185$.
78. Pinter M, Ulbrich G, Sieghart W, et al. Hepatocellular carcinoma: a phase II randomized controlled double-blind trial of transarterial chemoembolization in combination with biweekly intravenous administration of bevacizumab or a placebo. Radiology. 2015;277: 903-912.

79. Chisholm JC, Merks JHM, Casanova M, et al. Open-label, multicentre, randomised, phase II study of the EpSSG and the ITCC evaluating the addition of bevacizumab to chemotherapy in childhood and adolescent patients with metastatic soft tissue sarcoma (the BERNIE study). Eur J Cancer. 2017;83:177-184.

80. Yoh K, Hosomi Y, Kasahara K, et al. A randomized, double-blind, phase II study of ramucirumab plus docetaxel vs placebo plus docetaxel in Japanese patients with stage IV non-small cell lung cancer after disease progression on platinum-based therapy. Lung Cancer. 2016; 99:186-193.

81. Doebele RC, Spigel D, Tehfe M, et al. Phase 2, randomized, openlabel study of ramucirumab in combination with first-line pemetrexed and platinum chemotherapy in patients with nonsquamous, advanced/metastatic non-small cell lung cancer. Cancer. 2015;121: 883-892.

82. Garon EB, Ciuleanu T-E, Arrieta O, et al. Ramucirumab plus docetaxel versus placebo plus docetaxel for second-line treatment of stage IV non-small-cell lung cancer after disease progression on platinum-based therapy (REVEL): a multicentre, double-blind, randomised phase 3 trial. Lancet. 2014;384:665-673.

83. Petrylak DP, Tagawa ST, Kohli M, et al. Docetaxel as monotherapy or combined with ramucirumab or icrucumab in second-line treatment for locally advanced or metastatic urothelial carcinoma: an open-label, three-arm, randomized controlled phase II trial. J Clin Oncol. 2016; 34:1500-1509.

84. Petrylak DP, de Wit R, Chi KN, et al. Ramucirumab plus docetaxel versus placebo plus docetaxel in patients with locally advanced or metastatic urothelial carcinoma after platinum-based therapy (RANGE): a randomised, double-blind, phase 3 trial. Lancet. 2017;390: 2266-2277.

85. Tabernero J, Yoshino T, Cohn AL, et al. Ramucirumab versus placebo in combination with second line FOLFIRI in patients with metastatic colorectal carcinoma that progressed during or after first-line therapy with bevacizumab, oxaliplatin, and a fluoropyrimidine (RAISE): a randomised, double-blind, multicentre, phase 3 study. Lancet Oncol. 2015;16:499-508.

86. Moore M, Gill S, Asmis T, et al. Randomized phase II study of modified FOLFOX-6 in combination with ramucirumab or icrucumab as second-line therapy in patients with metastatic colorectal cancer after disease progression on first-line irinotecan-based therapy. Ann Oncol. 2016;27:2216-2224.

87. Mackey JR, Ramos-Vazquez M, Lipatov O, et al. Primary results of ROSE/TRIO-12, a randomized placebo-controlled phase III trial evaluating the addition of ramucirumab to first-line docetaxel chemotherapy in metastatic breast cancer. J Clin Oncol. 2015;33:141-148.

88. Yardley DA, Reeves J, Dees EC, et al. Ramucirumab with eribulin versus eribulin in locally recurrent or metastatic breast cancer previously treated with anthracycline and taxane therapy: a multicenter, randomized, phase II study. Clin Breast Cancer. 2016;16: 471-479.

89. Fuchs CS, Tomasek J, Yong CJ, et al. Ramucirumab monotherapy for previously treated advanced gastric or gastro-oesophageal junction adenocarcinoma (REGARD): an international, randomised, multicenter, placebo-controlled, phase 3 trial. Lancet. 2014;383:31-39.

90. Wilke H, Muro K, Van Cutsem E, et al. Ramucirumab plus paclitaxel versus placebo plus paclitaxel in patients with previously treated advanced gastric or gastro-oesophageal junction adenocarcinoma (RAINBOW): a double-blind, randomised phase 3 trial. Lancet Oncol. 2014;15:1224-1235.

91. Yoon HH, Bendell JC, Braiteh FS, et al. Ramucirumab combined with FOLFOX as front-line therapy for advanced esophageal, gastroesophageal junction, or gastric adenocarcinoma: a randomized, double-blind, multicenter phase II trial. Ann Oncol. 2016;27:2196-2203. 
92. Zhu AX, Park JO, Ryoo BY, et al. Ramucirumab versus placebo as second-line treatment in patients with advanced hepatocellular carcinoma following first-line therapy with sorafenib (REACH): a randomised, double-blind multicentre, phase 3 trial. Lancet Oncol. 2015;16:859-870.

93. Je Y, Schutz FA, Choueiri TK. Risk of bleeding with vascular endothelial growth factor receptor tyrosine-kinase inhibitors sunitinib and sorafenib: a systematic review and meta-analysis of clinical trials. Lancet Oncol. 2009;10:967-974.

94. Lyden D, Hattori K, Dias S, et al. Impaired recruitment of bone-marrowderived endothelial and hematopoietic precursor cells blocks tumor angiogenesis and growth. Nat Med. 2001;7:1194-1201.

95. Kaplan RN, Riba RD, Zacharoulis S, et al. VEGFR1-positive haematopoietic bone marrow progenitors initiate the pre-metastatic niche. Nature. 2005;438:820-827.

96. Al-Moundhri MS, Al-Shukaili A, Al-Nabhani M, et al. Measurement of circulating levels of VEGF-A, -C, and -D and their receptors, VEGFR-1 and -2 in gastric adenocarcinoma. World $J$ Gastroenterol. 2008;14: 3879-3883.
97. Li C, Liu B, Dai Z, Tao Y. Knockdown of VEGF receptor-1 (VEGFR-1) impairs macrophage infiltration, angiogenesis and growth of clear cell renal cell carcinoma (CRCC). Cancer Biol Ther. 2011;12:872-880.

98. Sandler AB, Schiller JH, Gray R, et al. Retrospective evaluation of the clinical and radiographic risk factors associated with severe pulmonary hemorrhage in first-line advanced, unresectable nonsmall-cell lung cancer treated with carboplatin and paclitaxel plus bevacizumab. $J$ Clin Oncol. 2009;27:1405-1412.

99. Hang XF, Xu WS, Wang JX, et al. Risk of high-grade bleeding in patients with cancer treated with bevacizumab: a meta-analysis of randomized controlled trials. Eur J Clin Pharmacol. 2011;67:613-623.

100. Zhu X, Tian X, Yu C, Hong J, Fang J, Chen H. Increased risk of hemorrhage in metastatic colorectal cancer patients treated with bevacizumab: an updated meta-analysis of 12 randomized controlled trials. Medicine (Baltimore). 2016;95:e4232. 


\section{Supplementary material}

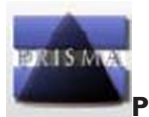

PRISMA 2009 Checklist

\begin{tabular}{llll}
\hline Section/topic $\quad \# \quad$ Checklist item & Reported \\
on page $\#$
\end{tabular}

Title

Title

I Identify the report as a systematic review, meta-analysis, or both.

I

Abstract

Structured summary

2 Provide a structured summary including, as applicable: background; objectives; data sources; study 2 eligibility criteria, participants, and interventions; study appraisal and synthesis methods; results; limitations; conclusions and implications of key findings; systematic review registration number.

\section{Introduction}

Rationale

Objectives

3 Describe the rationale for the review in the context of what is already known.

Methods

4 Provide an explicit statement of questions being addressed with reference to participants,

Protocol and registration

Eligibility criteria

Information sources

Search

Study selection

Data collection process

Data items

Risk of bias in individual studies

Summary measures Synthesis of results

Risk of bias across studies

Additional analyses

Results

Study selection

Study characteristics

Risk of bias within studies

Results of individual studies

Synthesis of results

Risk of bias across studies interventions, comparisons, outcomes, and study design (PICOS).

5 Indicate if a review protocol exists, if and where it can be accessed (eg, Web address), and, if available, provide registration information including registration number.

6 Specify study characteristics (eg, PICOS, length of follow-up) and report characteristics (eg, years considered, language, publication status) used as criteria for eligibility, giving rationale.

7 Describe all information sources (eg, databases with dates of coverage, contact with study authors to identify additional studies) in the search and date last searched.

8 Present full electronic search strategy for at least one database, including any limits used, such that it could be repeated.

9 State the process for selecting studies (ie, screening, eligibility, included in systematic review, and, 5 if applicable, included in the meta-analysis).

10 Describe method of data extraction from reports (eg, piloted forms, independently, in duplicate) 5 and any processes for obtaining and confirming data from investigators.

II List and define all variables for which data were sought (eg, PICOS, funding sources) and any 5 assumptions and simplifications made.

12 Describe methods used for assessing risk of bias of individual studies (including specification of 5 whether this was done at the study or outcome level), and how this information is to be used in any data synthesis.

13 State the principal summary measures (eg, risk ratio, difference in means).

14 Describe the methods of handling data and combining results of studies, if done, including measures of consistency $\left(\mathrm{eg}, \mathrm{l}^{2}\right)$ for each meta-analysis.

15 Specify any assessment of risk of bias that may affect the cumulative evidence (eg, publication bias, 5 selective reporting within studies).

16 Describe methods of additional analyses (eg, sensitivity or subgroup analyses, meta-regression), 5 if done, indicating which were pre-specified.

17 Give numbers of studies screened, assessed for eligibility, and included in the review, with reasons for exclusions at each stage, ideally with a flow diagram.

18 For each study, present characteristics for which data were extracted (eg, study size, PICOS, follow-up period) and provide the citations.

19 Present data on risk of bias of each study and, if available, any outcome level assessment (see item 12).

20 For all outcomes considered (benefits or harms), present, for each study: (a) simple summary data for each intervention group (b) effect estimates and confidence intervals, ideally with a forest plot.

21 Present results of each meta-analysis done, including confidence intervals and measures of consistency.

22 Present results of any assessment of risk of bias across studies (see item I5).

5

5

5

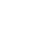

5


PरIS M

PRISMA 2009 Checklist (Continued)

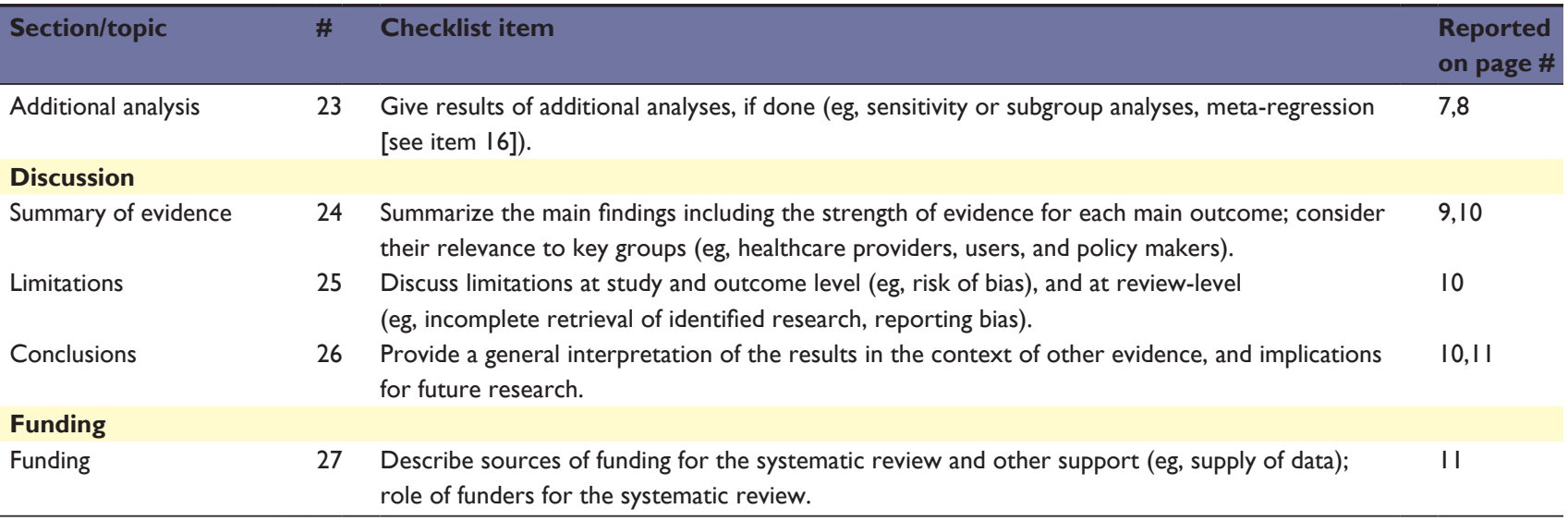

Notes: Moher D, Liberati A, Tetzlaff J, Altman DG; The PRISMA Group. Preferred Reporting Items for Systematic Reviews and Meta-Analyses: the PRISMA statement. PLoS Med. 2009;6(7):el000097. For more information, visit: www.prisma-statement.org.

\section{Publish your work in this journal}

OncoTargets and Therapy is an international, peer-reviewed, open access journal focusing on the pathological basis of all cancers, potential targets for therapy and treatment protocols employed to improve the management of cancer patients. The journal also focuses on the impact of management programs and new therapeutic agents and protocols on

\section{Dovepress}

patient perspectives such as quality of life, adherence and satisfaction. The manuscript management system is completely online and includes a very quick and fair peer-review system, which is all easy to use. Visit http://www.dovepress.com/testimonials.php to read real quotes from published authors. 